\title{
Adult age differences in familiarization to treadmill walking within virtual environments
}

\author{
Michael Schellenbach ${ }^{\mathrm{a}, \mathrm{b}, *}$, Martin Lövdén ${ }^{\mathrm{a}, \mathrm{c}}$, Julius Verrel ${ }^{\mathrm{a}}$, Antonio Krüger ${ }^{\mathrm{b}}$, Ulman Lindenberger ${ }^{\mathrm{a}}$ \\ ${ }^{a}$ Center for Lifespan Psychology, Max Planck Institute for Human Development, Berlin, Germany \\ ${ }^{\mathrm{b}}$ Institute for Geoinformatics, University of Münster, Münster, Germany \\ ' Department of Psychology, Lund University, Lund, Sweden
}

\section{A R T I C L E I N F O}

\section{Article history:}

Received 18 December 2008

Received in revised form 25 August 2009

Accepted 8 November 2009

\section{Keywords:}

Treadmill

Walking

Aging

Virtual environments

\begin{abstract}
A B S T R A C T
We assessed age-related differences in adults in familiarization to treadmill walking within virtual environments (VE), and examined whether treadmill walking after familiarization resembles overground walking. Seventeen younger and 17 older adults walked at preferred speed on an overground walkway and afterwards walked at the same speed for 20 min on a treadmill coupled to a VE. A motion capture system was used to measure spatio-temporal gait parameters. On the treadmill, both younger and older adults initially displayed decreased step length and increased step width, cadence, and time in double support relative to overground walking. Except for time in double support, step characteristics approached overground walking-behavior with a negatively accelerated trend. After 15 min of treadmill walking, changes became minor corresponding to less than $1 \%$ deviations to individuals' overground walking. At the end of familiarization, average differences in step length, cadence, and double support relative to overground walking were reduced to less than 5 percent in both age groups. For step width, younger adults approximated overground walking after 20 min more closely than older adults, probably reflecting larger initial differences between treadmill and overground walking among older adults. We conclude (a) that 20 min of familiarization to treadmill walking in a VE are sufficient to reach stable walking patterns resembling those observed in overground walking, but that some differences between the two settings remain, especially in older adults; (b) that sufficient familiarization to the treadmill is needed to ascertain the validity and generalizability of comparisons between younger and older adults.
\end{abstract}

(c) 2009 Elsevier B.V. All rights reserved.

\section{Introduction}

The use of treadmills, in clinical and research settings [1-6], rests upon the assumption that reliable and valid measures of gait can be obtained during treadmill walking (TW) and that TW can be generalized to overground walking (OW). TW, in theory, is mechanically equivalent to $\mathrm{OW}$, but in reality walking on a treadmill is initially an unfamiliar experience.

Recent studies $[7,8]$ report difficulties in familiarization to TW, especially for older adults. In these studies, participants were asked to walk for up to $15 \mathrm{~min}$ on a motorized treadmill equipped with handrails but not with visual flow. Participants decided themselves how long they needed handrails. After 6 min of TW and onwards, younger participants' mean spatio-temporal gait parameters, such

\footnotetext{
* Corresponding author at: Center for Lifespan Psychology, Max Planck Institute for Human Development, Lentzeallee 94, D-14195 Berlin, Germany. Tel.: +49 30 82406296; fax: +49308249939.

E-mail address: schellenbach@mpib-berlin.mpg.de (M. Schellenbach).
}

as step length (SL) and cadence, were not statistically different from OW. However, the walking behavior of older adults continued to differ from OW even after 14 min of walking, and most of them still held onto the handrails. The presence of handrails for safety reasons or as support for the initial familiarization process is common in these kind of studies. Reviewing the literature reveals that only studies with younger adults (e.g. [9]) have examined familiarization to TW without handrails. In order to better compare familiarization to TW across age groups we argue that handrail-use should be prevented for both age groups so that older adults possible bias for protecting gait stability does not reduce their amount of actual familiarization to free TW as compared with younger adults.

A VE with a treadmill-based walking interface has recently been developed in several research fields, both for clinical and motor studies and for simulators of real world scenarios. In these fields, research interests focus on the ecologically valid laboratory frameworks for prototyping and evaluating mobile assistive technologies, for example, for examining interactions between cognitive and motor tasks $[10,11]$. 
Adding a VE to TW has recently been reported to produce negative effects on walking behavior $[12,13]$. In these studies, participants walked on a treadmill with a VE-corridor projected onto a concave screen. This condition was compared to a non-VE condition, in which participants just saw a static picture of the corridor. In the VE, participants walked with shorter and wider steps, and displayed more within-subject variability in these measures. No direct comparison to OW was reported and participants were not familiarized with the setting. Notably, the VE used in this experiment induced the perception that participants were continuously and slowly turning. This property, rather than the VE per se, may have produced the observed effects. More elaborated virtual reality laboratories equipped with a treadmill are also trying to simulate ecologically valid walking in VE (e.g. $[14,15])$, but their focus has mainly been on the visual and motor perception of the simulated world. For example, Iwata [14] analyzed the motion data more precisely but validated his system with visitors only (without a standardized assessment). So far there are no experiments known that used the VE to support the familiarization of younger as well as older adults to TW, which is surprising because a VE may, if it is not inducing turning-motions, reduce potential negative effects on TW that stem from a static visual flow.

In this experiment we studied step-related variables during 20 min of familiarization to TW in a VE designed as a straight outdoor path with neighboring trees and benches, which allowed for distance estimation. For the participants, this corresponded to the experience of walking on a straight path without any degree of turning. The VE also contained static parts, such as a background sky, so that the participant could orient themselves relative to stable properties of the VE. We examined the similarity and

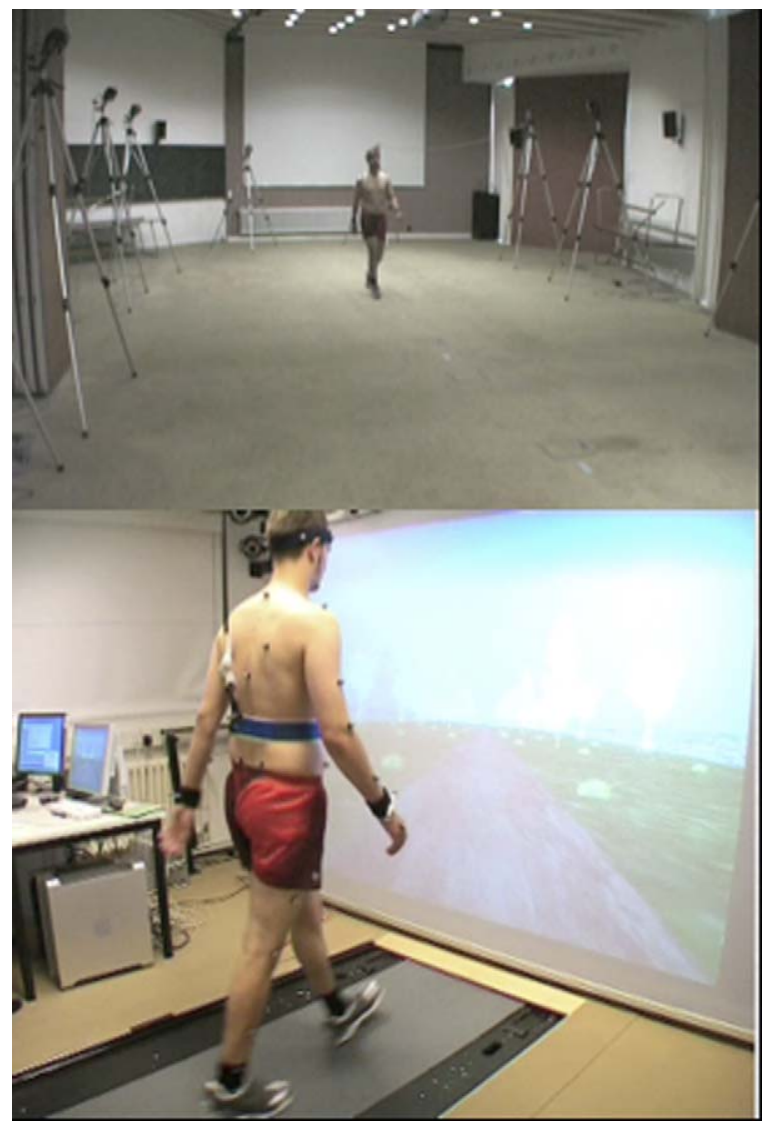

Fig. 1. Setting in overground walking session (top) and participant walking on the treadmill within a virtual environment (bottom). differences between initial TW, TW after familiarization, and OW. To force participants to familiarize to unconstrained walking, no handrail was provided, and the treadmill speed was adapted to individuals' average speed observed in the overground condition. As the treadmill used in our laboratory environment is at floor level, has no handrails, and was placed in front of a VE displaying the visual flow of a straight path (see Fig. $1 \mathrm{~b}$ ), we expected that TW after familiarization would more closely approximate OW than has been the case in earlier studies.

\section{Methods}

\subsection{Participants}

Seventeen younger adults $\left(M_{\text {age }}=23.9\right.$ years; $S D_{\text {age }}=2.73$ years; 10 women and 7 men $)$ and 17 older adults $\left(M_{\text {age }}=71.2\right.$ years; $S_{\text {age }}=2.02$ years; 7 women and 10 men) were recruited from the participant pool of the Max Planck Institute for Human Development, Berlin, Germany. We excluded individuals with conditions that are known to influence balance or gait performance. All participants had normal or corrected-to-normal vision and hearing, and little or no treadmill exposure. Also, participants in both age groups reported no fall within the last year.

Written consent was obtained prior to the experiment. Each participant received 20 Euros for participation. The Institute's Ethics Committee approved the study.

\subsection{Apparatus}

We used a 12-camera Vicon motion capture system (Vicon 612, Vicon Ltd., Oxford, UK), sampling at $200 \mathrm{~Hz}$, for recording participants' limb movements. Reflective markers were placed on relevant anatomical landmarks according to the VICON Plugin-Gait Model. Only spatio-temporal gait parameters entered the present analysis.

A 34-m walkway was used for the OW trials. Fig. 1a shows the setting of the overground session. Data were also captured on a motorized treadmill (Woodway $\mathrm{GmbH}$, Weil am Rhein, Germany), with the walking area $(200 \mathrm{~cm} \times 70 \mathrm{~cm})$ at the level of the surrounding floor. No handrail was present. For safety reasons, a harness was fastened around the waist of the participant and attached to the ceiling. In front of the treadmill, a $200 \mathrm{~cm} \times 270 \mathrm{~cm}$ screen was mounted. A VE consisting of a straight path was back-projected onto the screen, which immersed about $100^{\circ}$ of the participant's view. The visual flow of the VE was synchronized to the speed of the treadmill with an empirically established flow/speed ratio (see also [16]). Fig. 1b shows a participant walking on the treadmill.

\subsection{Design and procedure}

In a first session, OW was captured, with participants completing the treadmill condition together with cognitive tasks in a second session.

During OW, participants walked at self-selected speeds on a walkway, with stretches of straight paths connected by curves. Coordinates of the reflective markers were captured during $8 \mathrm{~m}$ of straight walking using the motion measurement system during 30 OW trials. Participants walked continuously; after each motion capture they walked back to the start and immediately continued with the next trial. There was a short break after the 10th and the 20th trial. Time and distance between the feet markers' appearance and disappearance in the motion capture range was used to calculate speed of walking. We averaged this speed over all thirty trials to arrive at an individualized measure of preferred overground speed.

During TW, the speed was set to each participant's average overground speed, as observed in the first session. Walking speed ranged from 3.4 to $5.9 \mathrm{~km} / \mathrm{h}$ $(\mathrm{M}=4.8 \mathrm{~km} / \mathrm{h} ; \mathrm{SD}=0.6 \mathrm{~km} / \mathrm{h})$ for the older adults and from 4.1 to $5.9 \mathrm{~km} / \mathrm{h}$ $(\mathrm{M}=4.9 \mathrm{~km} / \mathrm{h} ; \mathrm{SD}=0.6 \mathrm{~km} / \mathrm{h})$ for the younger adults; age-group differences in average walking speed were not significant, $t(31)=.433, p>.668$. Participants walked on the treadmill for $20 \mathrm{~min}$, with recording starting once the designated speed was reached. Participants had no information about the time passed.

\subsection{Data processing and statistical analyses}

The motion data (heel and toe markers) were post-processed in Matlab 7.4 (Mathworks, Natick, MA). From the motion data from each trial in the overground session, we used only the part where the subject was completely visible. In the treadmill session we split the data into 1-min intervals, extracting the gait cycles for all individuals from this data. Mean of SL, cadence, step width (SW), and percentage time in double support were the dependent variables, which were first computed separately for the left and right foot and then averaged over the feet. Finally, all trials of the overground condition were averaged.

As background information for the comparisons between OW and TW reported below, we addressed the effect of age and sex on OW with univariate 2 (Age Group: younger/older) $\times 2$ (Sex) ANOVAs separately for each dependent variable. A second set of ANOVAs included leg length and speed as covariates to address age and sex differences when these variables were statistically controlled. 
To compare OW and unfamiliar TW as function of age and sex, a set of mixed 2 (Age Group; younger/older) $\times 2$ (Sex) $\times 2$ (Condition; overground/first minute treadmill) ANOVAs were conducted separately for each dependent variable Condition was the within-subjects factor. Analogues ANOVAs were performed for more familiarized TW. Because we were mainly interested in the effects involving condition, which do not vary by speed and height, no covariates were included in this and the following models.

To estimate the familiarization function during TW and group differences in this function, we first, for each minute, computed each individual's percentage change from OW for a designated minute of TW [(treadmill - overground)/overground] Thus, a score of 0 for a given minute of TW means that values for treadmill and OW are identical. Then, we averaged the data into five-minute blocks and performed mixed 2 (Age Group; younger/older) $\times 2$ (Sex) $\times 2$ (Time; first/second/third/fourth five-minute block) ANOVA. Polynomial contrasts were inspected to detect linear and quadratic time effects. Additionally, we calculated regressions to fit quadratic cubic and exponential functions to the data. Only statistically significant timerelated effects are reported here.

\section{Results}

\subsection{Overground walking}

Statistical analyses revealed main effects of age for SL, cadence, and $\mathrm{SW}, \mathrm{Fs}(1,30)>4.80$, ps $<.036, \eta^{2} \mathrm{~s}>.134$. Younger adults walked with longer and wider steps and lower cadence. The main effect of sex was significant for SL and cadence, $F s(1,30)>5.10$, ps $<.031, \eta^{2} \mathrm{~s}>.141$. Men walked with longer steps and lower cadence than women. Means and standard deviations for leg length, speed, and the step-related dependent variables as functions of age and sex are reported in Table 1 .

After statistically controlling for leg length and speed, the main effect of age for SW, SL, and cadence remained significant, Fs $(1,28)>4.58, p s<.041, \eta^{2} s>.141$. Relative to their speed and leg length, younger adults walked with wider and longer steps, and with lower cadence. In addition, the main effect sex remained significant for SL and cadence, and a significant effect emerged for $\mathrm{SW}, \mathrm{Fs}(1,28)>4.37, \mathrm{ps}<.046, \eta^{2} \mathrm{~s}>.135$. Independent of their leg length and speed, men walked with longer and wider steps, and with lower cadence than women. No other effects were reliable. The observed pattern of age and sex effects for these step-related variables is fairly typical $[16,17]$.

\subsection{Overground and unfamiliarized treadmill walking}

As compared with OW, during unfamiliarized TW participants demonstrated an increase in $S W, F(1,30)=19.75, p<.001, \eta^{2}=.397$, cadence, $F(1,30)=31.53, p<.001, \eta^{2}=.245$, and time in double support, $F(1,30)=18.15, p<.001, \eta^{2}=.377$. SL also decreased, $F(1,30)=9.75, p=.004, \eta^{2}=.512$, on the treadmill (cf. Table 2 ). These effects did neither significantly interact with age nor with sex, but there was a trend of an age by condition interaction for cadence, $F(1,30)=3.32, p=.078, \eta^{2}=.100$. During the first minute on the treadmill older adults tended to increase their cadence more than younger adults. Main effects of age and sex revealed the same pattern of significant effects as those reported for overground walk.

\subsection{Patterns of 20-min treadmill walking}

Statistical analyses showed linear and quadratic time effects (across the four five-minute blocks) for all four variables, $F s(1,30)>4.84, p s<.036, \eta^{2} \mathrm{~s}>.139$. For SL, cadence, and SW, participants displayed initially rapid change towards the overground values, followed by a flattening out of the function (see Fig. 2a-c). After about $15 \mathrm{~min}$ of TW, remaining changes were small ( $<1 \%$ of overground behavior). In contrast, participants increased the time spent in double support relative to OW during the initial period of TW, and did not reliably decrease their double support thereafter (see Fig. 2d). For cadence, the main effects of time were qualified by a linear age by time interaction that was marginally significant, $F(1,30)=4.05, p=.053, \eta^{2}=.119$. Interestingly, older adults tended to show more rapid changes towards their overground behavior than younger adults.

For all sex and age groups and all gait parameters, the fitted linear plus quadratic as well as linear plus quadratic and cubic curves showed higher $r^{2}$-values than the exponential curves. Furthermore, with a few exceptions, adding a cubic trend did not lead to reliable increments in $r^{2}, p s>.16$. In the exceptions the additive cubic function only showed small increases for $r^{2}$-values, changes in $r^{2}<.16$. Therefore we used the linear plus quadratic function to further examine the time course of familiarization (see

Table 1

Means and standard deviations (SD) of gait parameters in overground walking.

\begin{tabular}{|c|c|c|c|c|c|c|c|c|}
\hline Age & Sex & & Step length & Cadence & Step width & Double support & Speed & Leg length \\
\hline \multirow[t]{4}{*}{ Younger } & Men & Mean & 803.86 & 107.24 & 71.15 & 56.97 & 5.19 & 915.71 \\
\hline & & SD & 79.02 & 8.03 & 25.84 & 1.89 & 0.56 & 47.21 \\
\hline & Women & Mean & 702.36 & 109.98 & 70.11 & 57.81 & 4.67 & 873.00 \\
\hline & & SD & 68.40 & 7.74 & 19.52 & 1.40 & 0.55 & 50.34 \\
\hline \multirow[t]{4}{*}{ Older } & Men & Mean & 706.70 & 109.81 & 63.35 & 57.94 & 4.69 & 923.00 \\
\hline & & SD & 77.55 & 4.77 & 12.41 & 1.25 & 0.64 & 50.56 \\
\hline & Women & Mean & 685.60 & 118.33 & 49.38 & 57.37 & 4.91 & 884.29 \\
\hline & & SD & 54.19 & 8.01 & 9.33 & 1.31 & 0.66 & 60.51 \\
\hline
\end{tabular}

Table 2

Means and standard deviations (SD) of gait parameters in first minute and last (20th) minute treadmill walking.

\begin{tabular}{|c|c|c|c|c|c|c|}
\hline Age & Sex & & Step length & Cadence & Step width & Double support \\
\hline \multirow[t]{4}{*}{ Younger } & Men & Mean & 779.38/803.49 & $114.22 / 111.59$ & $79.75 / 68.78$ & $57.23 / 58.21$ \\
\hline & & SD & $66.36 / 70.71$ & $5.97 / 5.41$ & $35.64 / 33.81$ & $1.55 / 2.14$ \\
\hline & Women & Mean & $698.87 / 729.33$ & $114.84 / 110.36$ & $82.33 / 69.47$ & $58.81 / 59.04$ \\
\hline & & SD & $66.94 / 72.18$ & $8.28 / 6.01$ & $20.10 / 22.47$ & $1.11 / 1.74$ \\
\hline \multirow[t]{4}{*}{ Older } & Men & Mean & $685.98 / 720.86$ & $118.60 / 112.19$ & $90.22 / 75.81$ & $59.05 / 59.19$ \\
\hline & & SD & $96.79 / 68.48$ & $5.81 / 5.27$ & $19.05 / 24.52$ & $1.50 / 1.41$ \\
\hline & Women & Mean & $648.77 / 703.41$ & $132.73 / 120.48$ & $62.48 / 58.42$ & $58.28 / 58.59$ \\
\hline & & SD & $73.97 / 50.00$ & $21.75 / 16.80$ & $30.56 / 24.34$ & $1.91 / 1.75$ \\
\hline
\end{tabular}



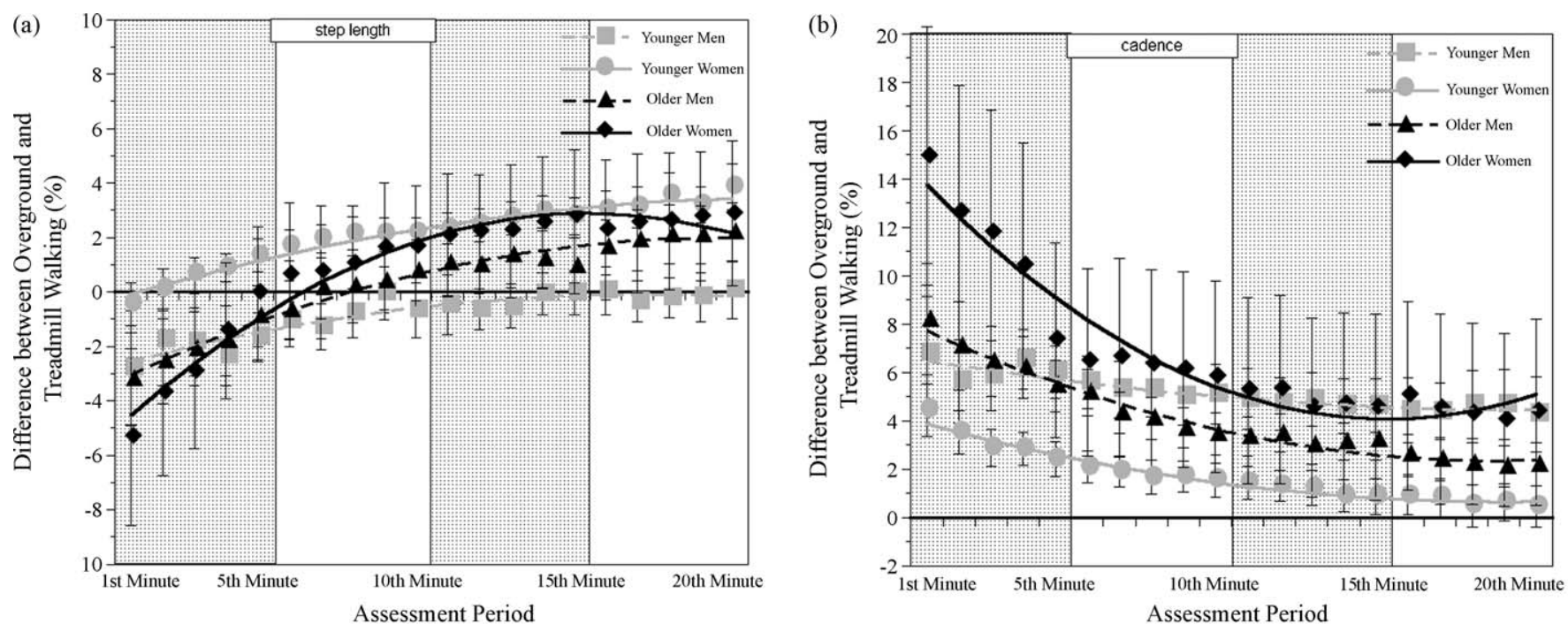

(c)
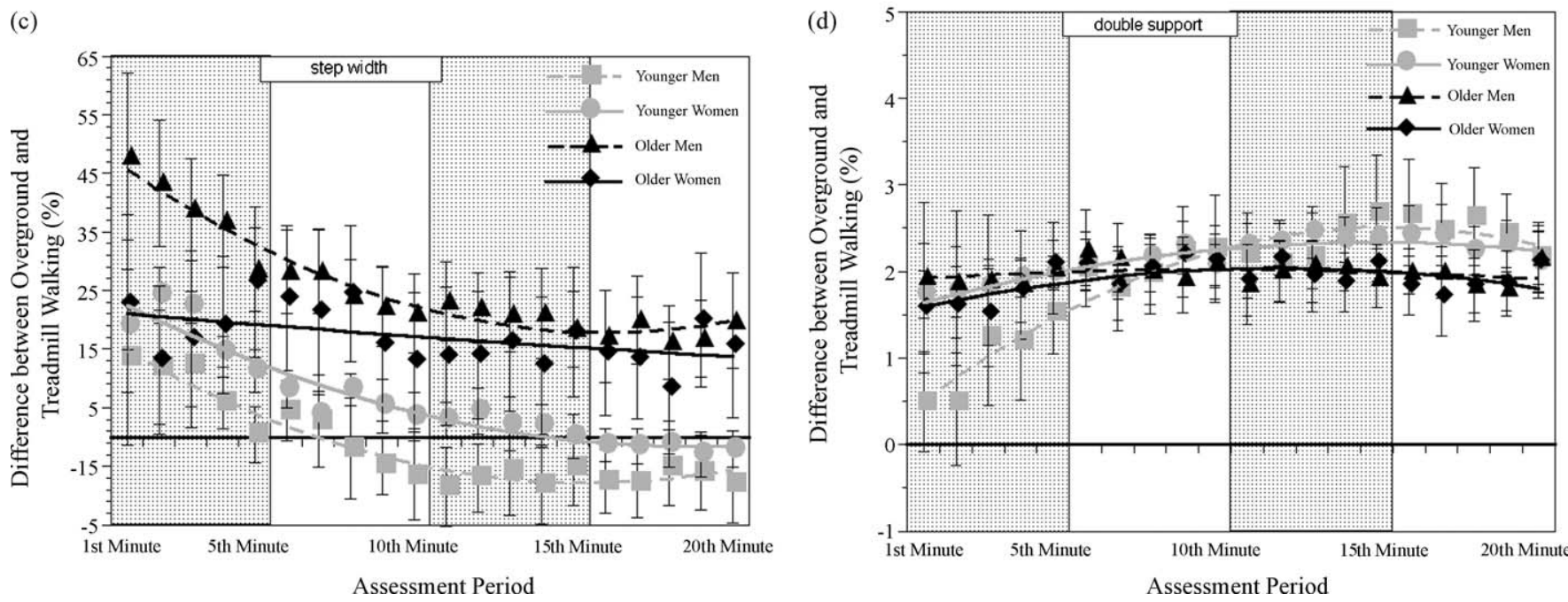

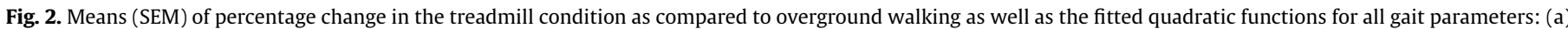
step length, (b) cadence, (c) step width, and (d) double support.

Fig. 2). Except for older women's SW, the fitted functions for SL, cadence, and SW reached their extreme value between 15 and 20 min, $r^{2}>.87, F(2,17)>54.78, p<.05$. SW in older women did not show reliable time-related trends. For double support, we observed maximum values between 14 and $16 \mathrm{~min}$ for younger adults, $r^{2}>.85, F(2,17)>46.89, p<.05$, and between 11 and $13 \mathrm{~min}$ for older women, $r^{2}=.426, F(2,17)=6.307, p<.05$.

\subsection{Overground and more familiarized treadmill walking}

In the last minute of treadmill, participants still walked with more time in double support, $F(1,30)=132.74, p<.001, \eta^{2}=.816$, and with higher cadence, $F(1,30)=6.04, p<.02, \eta^{2}=.167$, than for OW (cf. Table 2). However, these differences were rather small in magnitude $(<5 \%)$. Notably, participants also took longer steps (on average $2.5 \%$ ), $F(1,30)=8.10, p<.01, \eta^{2}=.213$, which is opposite to the shorter steps that they started out with on the treadmill. For SW, the age by condition interaction reached significance, $F(1,30)=6.51$, $p<.02, \eta^{2}=.178$. Younger adults showed smaller differences between OW and TW in SW than older adults (see Fig. 2). Similar but statistically not reliable trends were also observed for SL and cadence. Main effects of age and sex revealed the same pattern of significant effects as those found before for OW.

\section{Discussion}

Participants displayed relatively large deviations from their overground behavior when initially walking on the treadmill. SW showed the most dramatic differences, which might be interpreted as an effort to increase gait stability. The pattern of decreased SL and increased cadence and double support can be interpreted along similar lines. Though significant age and sex differences in this behavior did not materialize, the trends, especially for cadence, suggest this initial tendency to walk more carefully on the treadmill may be more pronounced in older adults than in younger adults. Nevertheless, we conclude that both younger and older adults both show deviations from normal OW during initial phases of TW (see also [7,8,18-20]).

Changes in the step-related measures in the course of the subsequent $20 \mathrm{~min}$ of TW were well captured by a quadratic function. After about 15 min of TW, further changes became minor ( $<1 \%$ of overground behavior), suggesting that participants' behavior was at or near asymptotic levels. In the sense of reaching a relatively temporally stable walking pattern, these results would seem to suggest that both younger and older adults can become familiar with TW within 20 min. However, relative to OW, small but reliable differences for SL, cadence, and double support remained, 
suggesting that the TW pattern asymptotes at a somewhat different level than OW. Percentage time in double support also did not return to overground values, but the remaining differences were again small (2\%) and did not differ by age group. In contrast, for SW, younger adults came closer to OW than older adults. Probably, this difference was primarily due to the large initial increase in SW relative to OW for the older adults (up to $50 \%$ increase).

In sum, all groups reached a relatively stable pattern of steprelated measures, suggesting that both younger and older adults can be familiarized to TW. At the same time, older adults did not fully abandon the more careful walking style that initially adopted when starting to walk on the treadmill.

In contrast to the studies of Wass and colleagues [7,8], we refrained from providing participants with the opportunity to use handrails, thereby forcing all participants to attempt to adjust to freely walking on the treadmill. Also, the treadmill velocity was set to individual participants' spontaneous overground speed. On the positive side, and in contrast to Wass and colleagues [8], these changes may have been the main reason why older persons showed clear signs of familiarization in the course of the present observation period of $20 \mathrm{~min}$. At the same time, walking without handrails may have led older adults to initially adopt a very cautious walking style, thereby reducing the likelihood of reaching a stable walking pattern that approximates oW even more closely. Hence, further studies may consider including an initial period of handrail use followed by a longer familiarization trial.

In previous studies [12,13], TW in a VE was associated with larger SW and reduced SL, potentially indicating more instability than in a non-VE situation. In these experiments the participants walked in a virtual moving corridor coupled to the treadmill. The scene was rotating in order to move the virtual corridor endlessly. While subjects had to walk straight on the treadmill, they likely perceived themselves as walking along a slightly curved path. As mentioned by the authors, the somatosensory and visual systems may have been providing partially conflicting reference frames for the control of gait. Because it is evident that VE can influence perception, these findings highlight the need for improving VE towards more ecologically valid scenarios in order to not disturb but support the perceptual flow while walking. Therefore, we programmed an outdoor scenario that supported distance estimations, and added a horizon as stable environmental feature. Future studies need to compare OW to TW with and without various versions of VEs in order to directly address whether better optimized VE may improve treadmill familiarization beyond the levels observed in this study.

In sum, we found that relatively stable walking patterns can be attained and relatively similar walking patterns can be observed for OW and TW when providing sufficient familiarization to TW in a VE. This finding holds for both younger and older adults. At the same time, some differences remain, and especially older adults continue to walk in a slightly more cautious manner after 20 min of TW than during OW.

\section{Acknowledgements}

This work was conducted as part of the Sensorimotor-Cognitive Couplings Project at the Center for Lifespan Psychology, Max
Planck Institute for Human Development, Berlin, Germany. We thank Julia Wolff and Gabi Faust for their help with data collection. For additional help in data preprocessing we want to thank Petra Bistrosch, Djamila Maleika, Nicole Malik, Katrin Sauck and Antje Ullrich.

\section{Conflict of interest statement}

None declared.

\section{References}

[1] Baker PA, Evans OM, Lee C. Treadmill gait retraining following fractured neck-offemur. Archives of Physical Medicine and Rehabilitation 1991;72(9):649-52.

[2] Hesse S. Treadmill training with partial body weight support after stroke: a review. Neurorehabilitation 2008;23(1):55-65.

[3] Klein RM, Potteiger JA, Zebas CJ. Metabolic and biomechanical variables of two incline conditions during distance running. Medicine and Science in Sports and Exercise 1997;29(December(12)):1625-30.

[4] McCain KJ, Pollo FE, Baum BS, Coleman SC, Baker S, Smith PS. Locomotor treadmill training with partial body-weight support before overground gait in adults with acute stroke: a pilot study. Archives of Physical Medicine and Rehabilitation 2008;89(4):684-91.

[5] Pearce ME, Cunningham DA, Donner AP, Rechnitzer PA, Fullerton GM, Howard $\mathrm{JH}$. Energy-cost of treadmill and floor walking at self-selected paces. European Journal of Applied Physiology and Occupational Physiology 1983;52(1):115-9.

[6] Shibata M, Shimura M, Shibata S, Wakamura T, Moritani T. Determination of the optimal walking speed for neural relaxation in healthy elderly women using electromyogram and electroencephalogram analyses. European Journal of Applied Physiology and Occupational Physiology 1997;75(3):206-11.

[7] Matsas A, Taylor N, McBurney H. Knee joint kinematics from familiarised treadmill walking can be generalised to overground walking in young unimpaired subjects. Gait \& Posture 2000;11(1):46-53.

[8] Wass E, Taylor NF, Matsas A. Familiarisation to treadmill walking in unimpaired older people. Gait \& Posture 2005;21(1):72-9.

[9] Riley PO, Paolini G, Della Croce U, Paylo KW, Kerrigan DC. A kinematic and kinetic comparison of overground and treadmill walking in healthy subjects. Gait \& Posture 2007 Jun;26(1):17-24.

[10] Lövdén M, Schäfer S, Pohlmeyer AE, Lindenberger U. Walking variability and working memory load in aging: a dual-process account relating cognitive control to motor control performance. Journal of Gerontology Psychological Sciences 2008;63:121-8.

[11] Lövdén M, Schellenbach M, Grossman-Hutter B, Krüger A, Lindenberger U. Environmental topography and postural control demands shape aging-associated decrements in spatial navigation performance. Psychology and Aging 2005;20(4):683-94.

[12] Hollman JH, Brey RH, Bang TJ, Kaufman KR. Does walking in a virtual environment induce unstable gait? An examination of vertical ground reaction forces. Gait \& Posture 2007;26(2):289-94.

[13] Hollman JH, Brey RH, Robb RA, Bang TJ, Kaufman KR. Spatiotemporal gait deviations in a virtual reality environment. Gait \& Posture 2006;23(4):441-4.

[14] Iwata $\mathrm{H}$. Walking about virtual environments on an infinite floor. In: Proceedings of the IEEE virtual reality; 1999.

[15] Mohler BJ, Thompson WB, Creem-Regehr SH, Willemsen P, Herbert L, Pick J, Rieser JJ. Calibration of locomotion resulting from visual motion in a treadmillbased virtual environment. ACM Transactions of Applied Perception 2007;4(1):4

[16] Oberg T, Karsznia A, Oberg K. Basic gait parameters-reference data for normal subjects, 10-79 years of age. Journal of Rehabilitation Research and Development 1993;30(2):210-23.

[17] Prince F, Corriveau H, Hebert R, Winter DA. Gait in the elderly. Gait \& Posture 1997;5(2):128-35.

[18] Lavcanska V, Taylor NF, Schache AG. Familiarization to treadmill running in young unimpaired adults. Human Movement Science 2005;24(August(4)):544-57.

[19] Taylor NF, Evans OM, Goldie PA. Angular movements of the lumbar spine and pelvis can be reliably measured after 4 minutes of treadmill walking. Clinical Biomechanics 1996;11(8):484-6.

[20] Van Ingen Schenau GJ. Some fundamental aspects of the biomechanics of overground vs. treadmill locomotion. Medicine and Science in Sports and Exercise 1980;12(4):257-61. 